\title{
CONF-9409/53-2
}

UCRL-JC-117553

PREPRINT

\section{Thin Aerogel Films for Optical, Thermal, Acoustic, and Electronic Applications}

Lawrence W. Hrubesh and John F. Poco

This paper was prepared for submittal to the

International Symposium on Aerogels 4

Berkeley, CA

September 19-21, 1994

RECEIVED

NOV 171995

OSTI

September, 1994

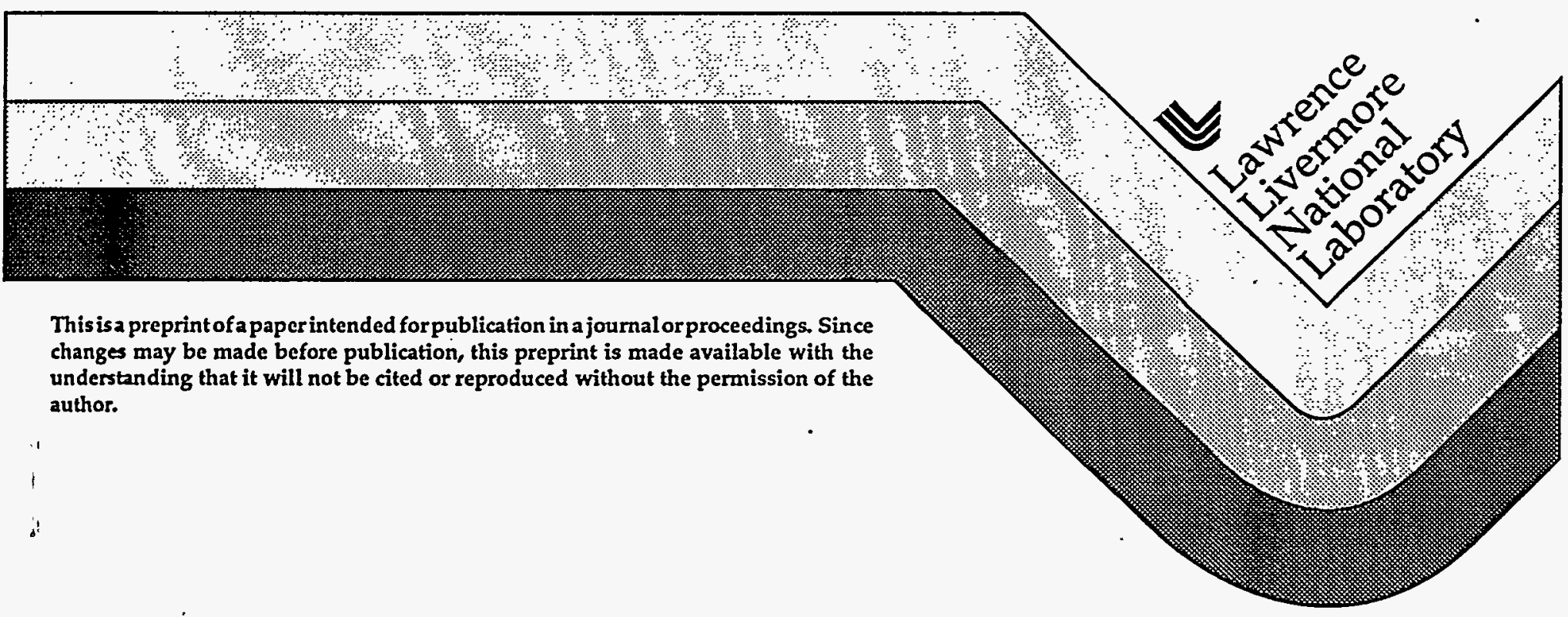


DISCLAIMER

This document was prepared as an account of work sponsored by an agency of the United States Government. Neither the United States Government nor the University of California nor any of their employees, makes any warranty, express or implied, or assumes any legal liability or responsibility for the accuracy, completeness, or usefulness of any information, apparatus, product, or process disclosed, or represents that its use would not infringe privately owned rights. Reference herein to any specific commercial product, process, or service by trade " name, trademark, manufacturer, or otherwise, does not necessarily constitute or imply its endorsement, recommendation, or favoring by the United States Government or the University of California. The views and opinions of authors expressed herein do not necessarily state or reflect those of the United States Government or the University of California, and shall not be used for advertising or product endorsement purposes. 


\title{
Thin aerogel films for optical, thermal, acoustic, and electronic applications*
}

\author{
L.W. Hrubesh and J.F. Poco \\ Chemistry and Material Sciences Department \\ Lawrence Livermore National Laboratory \\ Livermore, CA 94550
}

Abstract

Aerogels are a special class of continuously porous-solid materials which are characterized by nanometer size particles and pores. Typically, aerogels are made using sol-gel chemistry to form a solvent filled, high porosity gel that is dried by removing the solvent without collapsing the tenuous solid phase. As bulk materials, aerogels are known to have many exceptional, and even some unique physical properties. Aerogels provide the highest thermal insulation and lowest dielectric constant of any other material known. However, some important applications require the aerogels in the form of thin films or sheets. For example, electronic applications require micrometer thin aerogel films bonded to a substrate, and others require thicker films, either on a substrate or as free standing sheets. Special methods are required to make aerogel thin films or sheets. In this paper, we discuss the special conditions needed to fabricate thin aerogel films and we describe methods to make films and thin sheets. We also give some specific applications for which aerogel films are being developed.

*Work performed under the auspices of the U. S. Department of Energy by the Lawrence Livermore National Laboratory under Contract No. W-7405-ENG-48 


\section{Introduction}

Aerogels are unusual porous materials because of their unique microstructure consisting of pores and particles which are in the nanometer size range. Aerogels are typically made using sol-gel chemistry to form solvent filled gels which are dried in a way that does not shrink or collapse the weak solid structure of the solid matrix. The dried aerogel is an air filled porous solid, usually in monolithic or granular form. As bulk materials, aerogels are known to have many exceptional and even some unique physical properties which account for the large number and variety of potential applications for them. The exceptional optical, thermal, acoustical, and electronic properties of aerogels which result from their unique microstructure, have been well established and documented, particularly in the proceedings of the three prior International Symposia on Aerogels [1-3]. Most of the properties which are characteristic for bulk aerogels are also exhibited in other forms of the material, e.g., in thin sheets or films. A growing number of important applications require the aerogels either as free standing sheets or as thin layers on other substrate materials. Fabricating the aerogels in such forms presents problems due to the weak, tenuous nature of the highly porous aerogels and because special requirements are necessary to form and dry them. For purposes of discussion in this paper, we arbitrarily refer to the gels as aerogels if their porosity is $>75 \%$, xerogels if their porosity is $<75 \%$, and dense films if the porosity is $<1 \%$.

Sol-gel processes have been well developed, especially during the past decade or so, to produce advanced glasses, glass-ceramics, and ceramics, in both bulk and film forms. During this period, there have been several symposia on the subject and many journal articles have been published. There are good books and articles which describe the advantages, details, and applications of the sol-gel processes [4-6] and there are several review articles specific to sol-gel films [7-9]. An excellent source of information for all aspects of sol-gel processing of films, including porous films, is found in Brinker and Sherer's book, Sol-Gel Science, Chaps. 13 and 14 [6]. Most of the literature on sol-gel films deals with applications for full density coatings or partially densified films, such as AR coatings on optics. Very little work has been reported for processing porous sol-gel films where the films are to remain porous for their application. There are some articles which discuss sol-gel films up to about 50\% porosity [10-12] and one article discusses the preparation of a true aerogel film but the porosity is not reported [13]. In this paper, we discuss the special conditions and requirements which are needed to make highly porous aerogel films, i.e., porosity $>75 \%$.

Most of the methods already developed for applying sol-gel thin films and coatings generally apply, with modifications, to making true aerogel films as long as special consideration is given to prevent rapid drying by evaporation during deposition. In this paper we describe 
several methods that have been successfully used to form thin aerogel sheets and films. Measurements of properties which are important for various applications and the applications themselves will also be discussed.

\section{Experimental Procedures}

The methods used to make thin aerogel films depend on the thickness desired and whether or not the film is to be bonded to a surface. However, all of these methods have the common requirement that the gel must be formed under conditions in which the rate of evaporation is limited, both during and after the gel formation. To facilitate this we perform the coating processes within an enclosure that is maintained saturated with the vapor of the working solvent. The enclosure atmosphere also contains a partial pressure of ammonium hydroxide which helps to catalyze the gelation of the films. The most common aerogel films are silica, but we have also demonstrated our method with other metal oxides for which hydrolysis/condensation of the metal alkoxide is the predominant chemistry (e.g., zirconia, alumina, and tantala.) Generally, depending on the desired porosity of the final aerogel, we used two methods to prepare the precursor solution for the process. For gel porosities $>95 \%$ we used the two-step, partial hydrolysis/condensation chemistry reported previously for silica aerogels [14]. For gels with other porosities, we prepared a single-step base-catalysed hydrolysis solution according to the typical method for silica as follows: mix tetramethoxysilane (TMOS), water, methyl alcohol, and ammonium hydroxide, in a molar ratio of 1:2:4:0.01. An additional amount of alcohol is added to this mixture to establish the ultimate porosity of the gel. We discuss the following four processes which we have used to make high porosity films; dip coating, spinning, spray coating, and capillary film formation.

\subsection{Surface preparation}

From experience we found that most of the metal oxide gels adhere to glass or oxidized surfaces (probably through metal-oxide-metal bonds) but gels did not stick well to unprepared metal surfaces. Bonding to all oxidized surfaces was enhanced by etching the surface with a mild alkaline solution (e.g, $\mathrm{KOH}$ ), then rinsing with alcohol immediately prior to film deposition. For the opposite case of non-bonding we treated the surfaces with trimethylchlorosilane, to give a hydrophobic surface that serves as a release agent. 


\subsection{Dip coating}

Dip coating is the simplest of the coating processes, but it is used only when all surfaces of a substrate material are to be coated. With this method, film thicknesses less than a few micrometers are obtainable depending on the viscosity of the precursor and the withdrawl rate. In our work, the precise thickness was not an important parameter, so the dipping and withdrawing procedure was performed manually without concern for controlling the rates. Typical substrates were Pyrex glass slides of various sizes. These were simply dipped into the prepared precursor solution, withdrawn, then placed edgewise and vertical in a holder which is located within the enclosure. The time necessary for a gel film to form was found by trial to be only a few minutes. After the film is gelled, the entire holder containing the slides is immersed in a beaker of solvent and it remains surrounded by liquid until ready for supercritical drying.

\subsection{Spin coating}

This process is essentially the same as that used to spin glass coatings for electronic applications, except that it is performed with the spin apparatus entirely within an enclosure which has a solvent saturated atmosphere. Film thicknesses are typically less than two micrometers. Special modifications were performed on the apparatus to prevent the possibility of an explosive hazard. Typical substrates are Pyrex glass slides and silicon wafers up to 3" diameter. The procedure for forming films is to meter a droplet of precursor solution onto the spinning substrate while its spin rate is increasing up to a maximum speed of 1850 RPM. The spinner is immediately turned off and stopped with a brake, so that the sample is subject to minimal loss of solvent during gelation. Typically, the gel will form within a few minutes, after which the substrate is manually removed from the spin apparatus and immersed in solvent. The substrates with films are stored submersed in solvent until ready for supercritical drying.

\subsection{Spray coating}

This process has been used to put thicker single layer coatings on substrates like glass and silicon wafers. Films as thick as 80 micrometers have been acheived by this method. An aspirator is used to spray the precursor solution directly onto the substrate which is supported in a nearly vertical position within the enclosure. Excess solution drains by gravity, leaving a thick film which gels within a few minutes. These films have a varying thickness due to the draining, but the surface of the gel is smooth and continuous. After gelation, the substrate is manually immersed in solvent until ready for supercritical drying. 


\subsection{Surface tension coating}

This process is used to form uniform, relatively thick planar films on glass and.silicon substrates. Aerogel films with thicknesses from 2-50 micrometers have been achieved. Evaporation is essentially eliminated by using this method and therefore it does not require the special enclosure. This method is similar to dip coating but utilizes surface tension to draw the liquid onto the solid surfaces. This is best accomplished by using a fixture made by forming a space between the substrate to be coated and another flat surface. The surface of the flat is treated with a release agent to prevent bonding to the gel so that it is removable after the drying process. Spacers (shims or microspheres) are used to separate the substrates by the desired film thickness, then they are clamped together. The space between the substrates forms an effective capillary. Liquid precursor fills the available volume by capillarity when the fixture is dipped into the solution. The filled fixture is either be left immersed within the precursor solution to be encapsulated by the gel, or it is removed prior to gelation and placed in a solvent saturated atmosphere.

\subsection{Supercritical drying.}

All of the prepared gels were converted to aerogels using supercritical drying methods in order to prevent densification of the films. Either direct supercritical extraction (SCE) of the solvent was done at high temperature or an alternate low temperature extraction of carbon dioxide was performed after exchange of the original solvent. The method of drying chosen depended on the temperature stability of the substrate material. Gels on glass substrates were generally compatible with high temperature SCE whereas silicon wafers required a protective coating of silicon dioxide to survive the high temperature SCE of solvents. The procedure for direct SCE is to place the glass:container holding the submersed (or encapsulated) samples directly into an autoclave. The autoclave is filled with additional solvent (usually, alcohol) and sealed. The temperature of the autoclave is raised at a rate of $0.3^{\circ} \mathrm{C} / \mathrm{min}$. while the pressure increases to about 120 bars and excess pressure above that is released. After the temperature reaches about $280^{\circ} \mathrm{C}$, the pressure is released from the vessel at a controlled rate of $0.3 \mathrm{bar} / \mathrm{min}$. until a pressure of about 1.5 bar is reached. The autoclave is then purged with air as the vessel cools. This cycle typically takes 24 hours. Alternatively, a similar cycle is performed at temperatures less than $45^{\circ} \mathrm{C}$ after the solvent in the gel-film is first exchanged with liquid carbon dioxide. The exchange time to remove solvent from the thin films less than 50 micrometers thick was typically 2 hours. 


\subsection{Metallization}

Metallization of the aerogel was done by vapor or sputter deposition directly onto the asprepared aerogel film surfaces. For some cases, low resolution patterning was achieved by depositing the metal through an appropriate mask. Photo resist patterning techniques were applied only after the aerogel surface was coated with approximately $1000 \AA$ of parylene, to seal the pores of the aerogel.

\subsection{Characterization}

The morphology of the films and the film-substrate interface regions were examined using a high resolution $(\approx 20 \AA)$, field emission, scanning electron microscope (Hitachi S800).

The film or sheet thicknesses were measured using a Sloan DecTac II-A Profilometer. The DecTac II-A instrument measures thicknesses in the range 0.02 to 65 micrometers, with a vertical resolution of 0.5 nanometers, for a scan length up to 3 millimeters. The accuracy for micrometer thickness measurements with this instrument is better than $2 \%$.

Film adherence on glass and silicon wafers was qualitatively determined using a tape stick test with Scotch ${ }^{\otimes}$ tape. The tape was lightly pressed onto a surface of aerogel covering at least $1 \mathrm{~cm}^{2}$ area, then peeled off. The tape is examined for residue to determine if the aerogel has been lifted off or if the adhesive has been removed from the tape.

Refractive indexes of films were measured using an AutoEL ${ }^{\circledR}$-II Automatic Ellipsometer. The thickness measured by the DekTac profilometer is entered as input to the ellipsometer to calculate the refractive index of a film. Generally, it is difficult to apply this technique because the surface reflectivity of aerogels is typically less than $1 \%$ at a wavelength of $632.8 \mathrm{~nm}$. However, sufficient reflectivity is usually obtained at shallow angles of incidence (i.e., $<45^{\circ}$ ). Other physical properties of the aerogel films were determined from this measurement as follows:

The film densities were determined from the measurement of the optical refractive index, $n$, using the relation, $\rho=(n-1) / .209$, reported by Henning and Svensson for silica aerogels [15]. The dielectric constant and the porosity of the films were determined indirectly from the density, after measurement of the optical refractive index. The porosity is determined from the relation: $\Pi$ $=1-\rho / \rho s$ where $\rho s$ is the density of the solid. So for silica aerogel, with $\rho_{s}=2.19 \mathrm{~g} / \mathrm{cc}, \Pi=$ $[(1.458-n) / .458]$ is the percent porosity. Similarly, the dielectric constants for the silica aerogel films or sheets were determined after measurement of the optical refractive index using: $\kappa^{\prime}=1+1.6 p[16]$, so $\kappa^{\prime}=1+7.7(n-1)$. 


\section{Results}

Using the methods described, we have successfully fabricated thin, flat, uniform silica aerogel films, having various porosities, with measured thicknesses from less than 1 to 20 micrometers on glass and silicon wafer substrates. The adherence of the films to the substrates has been qualitatively determined by a tape stick test. Generally, good film adherence was obtained for all aerogels with a porosity less than about $86 \%$ and mixed results were observed for porosities between $86 \%$ and $95 \%$. For porosities $>95 \%$, the results were invalid because the aerogel is too weak to survive the application of the tape. We have also fabricated unattached aerogel sheets with measured thicknesses between 30 and 100 micrometers using the capillary fill method. These films and sheets have been examined using SEM to verify their aerogel-like structure. As an example of a typical film, figure 1 shows a series of SEMs of a 44 micrometer thick aerogel film deposited on a $7.6 \mathrm{~cm}$ diameter silicon wafer. The measured refractive index of this film is 1.049 , so its calculated density is about $0.235 \mathrm{~g} / \mathrm{cc}$, its porosity is about $89 \%$, and its dielectric constant is 1.38 . The film thickness uniformity over the full $7.6 \mathrm{~cm}$ diameter measured by the DekTac, was within the accuracy of the instrument (i.e., 2\%).

We have measured the refractive indexes of these films and sheets and we have calculated their bulk densities to confirm that they are aerogels having densities in the range from about $0.04-0.46 \mathrm{~g} / \mathrm{cc}$, and porosities in the range from $77 \%$ to $98 \%$. We have also calculated the dielectric constants of these films based on the measured refractive indexes. The dielectric constants span the range from about 1.06 to 1.7. Table 1 shows characteristic data for some representative thin aerogel films.

We developed processes to seal, pattern, and metallize thin aerogel films. An example of a high resolution pattern of gold metal on an aerogel film is shown in figure 2. This pattern of 4 micrometer wide strips was achieved with the following steps: 1) fabricate a 10 micrometer thick silica aerogel film on a $7.6 \mathrm{~cm}$ diameter silicon wafer using capillary filling and supercritical drying, 2) vapor deposit $\approx 100 \mathrm{~nm}$ parylene onto aerogel film, 3) vapor deposit $40 \mathrm{~nm}$ titanium metal then $500 \mathrm{~nm}$ gold, 4) spin on $1500 \mathrm{~nm}$ photoresist and dry at $90^{\circ} \mathrm{C}, 5$ ) mask and expose to ultraviolet, 6) remove mask, wash with $\mathrm{KOH}, 7$ ) etch away gold, 8) plasma etch titanium down to parylene, 9) ash away the remaining resist, leaving metallized pattern on parylene coated aerogel.

We have also sputtered thin $(<0.5$ micrometer) metal layers directly onto the aerogel surfaces and electroplated thicker $(>1.0$ micrometer) layers on the sputtered layers. 


\section{Discussion}

Aerogel films can serve in many useful applications. Here we describe several specific applications for which we have processed thin aerogel films. In most cases, the aerogel films are used on prototypes which are currently being tested for functionality and performance.

\subsection{Optical}

Thick aerogel films $(\approx 0.2$ millimeter) have been fabricated for use as coverslips on solar cells. In this case the aerogel films are formed, then transferred to the solar cell surface and bonded to it, instead of being formed directly on it. By replacing the high index glass coverslips normally used, the low refractive index aerogel causes less reflective Fresnel loss for incident light at its outer surface, allowing more light to reach the active surface, thus increasing the solar cell efficiency.

Thin aerogel films ( $\approx 30$ micrometers) have been deposited as a cladding on the outside of glass laser pump tubes. In this application, the aerogel acts as a refractive index match between the pump tube glass and air, to minimize reflection of internally generated light at the outer envelop of the tube, thus increasing the amount of excitation light reaching the lasing medium. The aerogel is preferred to antireflection coatings in this application because it provides a better match for a broader band of light waves.

\subsection{Thermal}

Thin aerogel films ( $\approx 25$ micrometers) have been formed on glass substrates for use in "cool" IR detectors. Here, the aerogel film serves as a thermal barrier to shield the IR detector elements from the heat radiated by the substrate materials.

Many thick sheets $(\approx 1$ millimeter) of aerogel are coated with thin layers of metal, then laminated together to form a super-insulating thermal heat shield block.

\subsection{Acoustic}

Thick aerogel films $(\approx 0.5$ millimeter $)$ are formed on the suface of ceramic transducers to serve as acoustic impedance matching layers. Sending and receiving airborne acoustic waves is substantially increased by using aerogels in this application. 


\subsection{Electronic}

Thin aerogel films $(\approx 2$ micrometers) are formed on silicon wafers to provide a low dielectric constant substrate in integrated circuits. The dielectric constant for all aerogels is less than 2.0 and its value depends on the porosity, a controllable parameter for aerogel films. Dielectric constants of the aerogels well below 2.0 will enable significant improvements in the speed of integrated circuits.

Several thick organic aerogel films $(\approx 0.5$ millimeter $)$ are formed by capillary fill, then pyrolyzed to carbon aerogel films for use in aerocapacitors. These devices can provide specific capacitances in excess of 45 Farads per gram of material [17].

\subsection{Other applications}

Other applications in the microelectronics and electro-optics industries include high-speed electronic conductors for both Ultra Large Scale Integrated Circuits and for interconnections between computer chips.

Applications for the thin film dielectrics include:

- microwave striplines

- microwave circuits such as used in radars and communications

- low capacitance chip connectors

- extremely lightweight electronic packages

- power transmission high voltage insulators

- spacers for electrodes in vacuum tubes

Some other applications of thin aerogel films include:

- membranes for gas separations

- flat panel displays

- window glazing

- electrical standoffs

- light bulbs for diffusing light

- passivation layer for oxidative metals

- insulative layer between stacks of metals or polymers

- anti-reflective films 
- cladding on optical fibers

- optical, chemical sensors

This is only a partial listing of potential applications for aerogel films. High porosity films appear to have as many applications as the bulk aerogels do. However in both cases, continued development is needed to refine the processes, to reduce processing costs, and to demonstrate the practical uses of the technology. In the case of thin aerogel films, the effort has just begun.

\section{Conclusions}

We have shown that it is possible and practical to form highly porous, true aerogel films on substrates using a variety of common deposition methods, if care is taken to slow evaporation of solvents during gelation, and if supercritical drying is done to preserve their tenuous structure. The aerogel films are good quality, bond well to the substrates, and are strong enough to survive other processing steps used to prepare them for specific applications. Most importantly, the aerogel films exhibit many of the exceptional properties which are characteristic of the bulk material and therefore they can be considered for use in a variety of optical, acoustical, thermal, and electronic applications. We anticipate that with further development, aerogel films and sheets will be be used in such applications in the near future.

\section{References:}

1) Aerogels, Springer Proc. in Physics, edited by J. Fricke (Springer-Verlag, Heidelberg, Germany, 1986), Vol. 6.

2) Revue de Physique Apliquee', edited by R. Vacher, J. Phalippou, J. Pelous, and T. Woignier (Proc. 2nd Int. Symp. Aerogels 24, C4, Les Editions de Physique, Les Ulis Cedex, 1989).

3) Aerogels 3, Proc. 3rd Int'l. Symp. Aerogels, edited by J. Fricke (Elsevier Science Publishers, North Holland, 1992) (J. Non-Cryst. Solids 145-146, 1992).

4) D.R. Ulrich, J. Non-Cryst. Solids 100 (1988) 174.

5) Sol-gel Technology for Thin Films, Fibers, Preforms, Electronics and Specialty Shapes, edited by L.C. Klein (Noyes, Park Ridge, N.J., 1988).

6) C.J. Brinker and G.W. Sherer, Sol-Gel Science (Academic Press, N.Y., 1990). 
7) H. Dislich, in: Sol-gel Technology for Thin Films, Fibers, Preforms, Electronics and Specialty Shapes, ed. L.C. Klein (Noyes, Park Ridge, N.J., 1988) p. 50.

8) A.J. Hurd, in: Critical Reviews in Surface Chemistry, 1992.

9) C.J. Brinker, A.J. Hurd, P.R. Shrunk, G.C. Frye and C.S. Ashley, J. Non-Cryst. Solids 147 (1992) 424.

10) C.J. Brinker, A.J. Hurd, K.J. Ward, in: Ultrastructure Processing of Advanced Ceramics, eds. J.D. Mackenzie and D.R. Ulrich (Wiley, New York, 1988) p. 223.

11) H. Schmidt, G. Rinn, R. Nass and D. Sporn, in: Bett. Ceram. for Chem. III, eds. C.J. Brinker, D.E. Clark and D.R. Ulrich (Mat. Res. Soc., Pittsburgh, 1988) p.743.

12) H. Hirashima and K. Sudoh, J. Non-Cryst. Solids 145 (1992) 51.

13) R. Sempere', D. Bourret, A. Sivade, R. Etienne and J. Bouaziz, J. Non-Cryst. Solids 148 (1992) 499.

14) L.W. Hrubesh, T.M. Tillotson, and J.F. Poco, in: Chemical Processing of Advanced Materials, eds. L.L. Hench and J.K. West (Wiley, New York, 1992) p. 19.

15) S. Henning and L. Svensson, Phys. Scripta 23 (1981) 697.

16) L.W. Hrubesh, L.E. Keene, and V.R. Latorre, J. Mater. Res. 8 (1993) 1736.

17) S.T. Mayer, R.W. Pekala and J.L. Kaschmitter, J. Electrochem. Soc., 140 (1993) 446. 


\section{Figure Captions:}

Figure 1. SEMs of a typical silica aerogel film on a silicon substrate, prepared by the capillary fill method. The flat and uniformly thick aerogel film is shown bonded to a thin $\approx 1.5$ $\mu \mathrm{m})$ layer of silicon dioxide which was vapor deposited on the silicon wafer. The granular appearance of the aerogel is an artifact due to charging by the beam and its surface is actually much smoother than it appears.

Figure 2. Photograph of 4 micrometer wide gold metal strips patterned on a thin silica aerogel film $(\approx 1.3 \mu \mathrm{m})$ which is on a silicon wafer. This pattern was produced by photoresist processing after the aerogel was first coated with about $100 \mathrm{~nm}$ thick parylene polymer which remains after the process.

Table I. Characteristic data for some representative thin aerogel films on glass

\begin{tabular}{lccccc}
\hline Film type & Method & $\begin{array}{c}\text { Ave. thickness }(\mu \mathrm{m}) \\
\text { (measured) }\end{array}$ & $\begin{array}{c}\text { Refractive index } \\
\text { (measured) }\end{array}$ & $\begin{array}{c}\text { Porosity (\%) } \\
\text { (calculated) }\end{array}$ & $\begin{array}{c}\text { Diel. constant } \\
\text { (calculated) }\end{array}$ \\
\hline Silica & capillary & 18.1 & 1.062 & 86 & 1.48 \\
Silica & spin & 3.4 & 1.103 & 78 & 1.79 \\
Silica & spray & 44.1 & 1.049 & 89 & 1.38 \\
Zirconia & capillary & 24.7 & 1.059 & 95 & $-\ldots$ \\
Silica & capillary & 113.5 & 1.009 & 98 & 1.07 \\
\hline
\end{tabular}



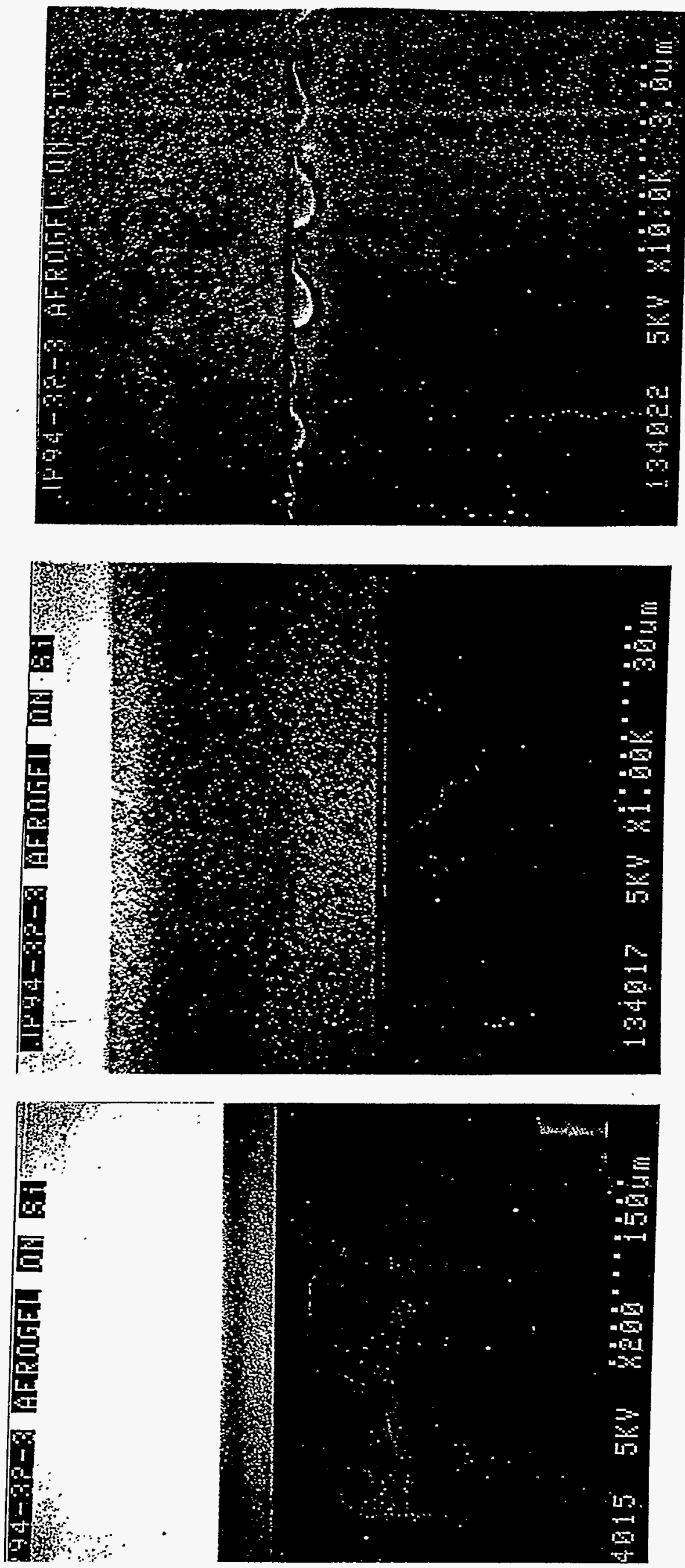


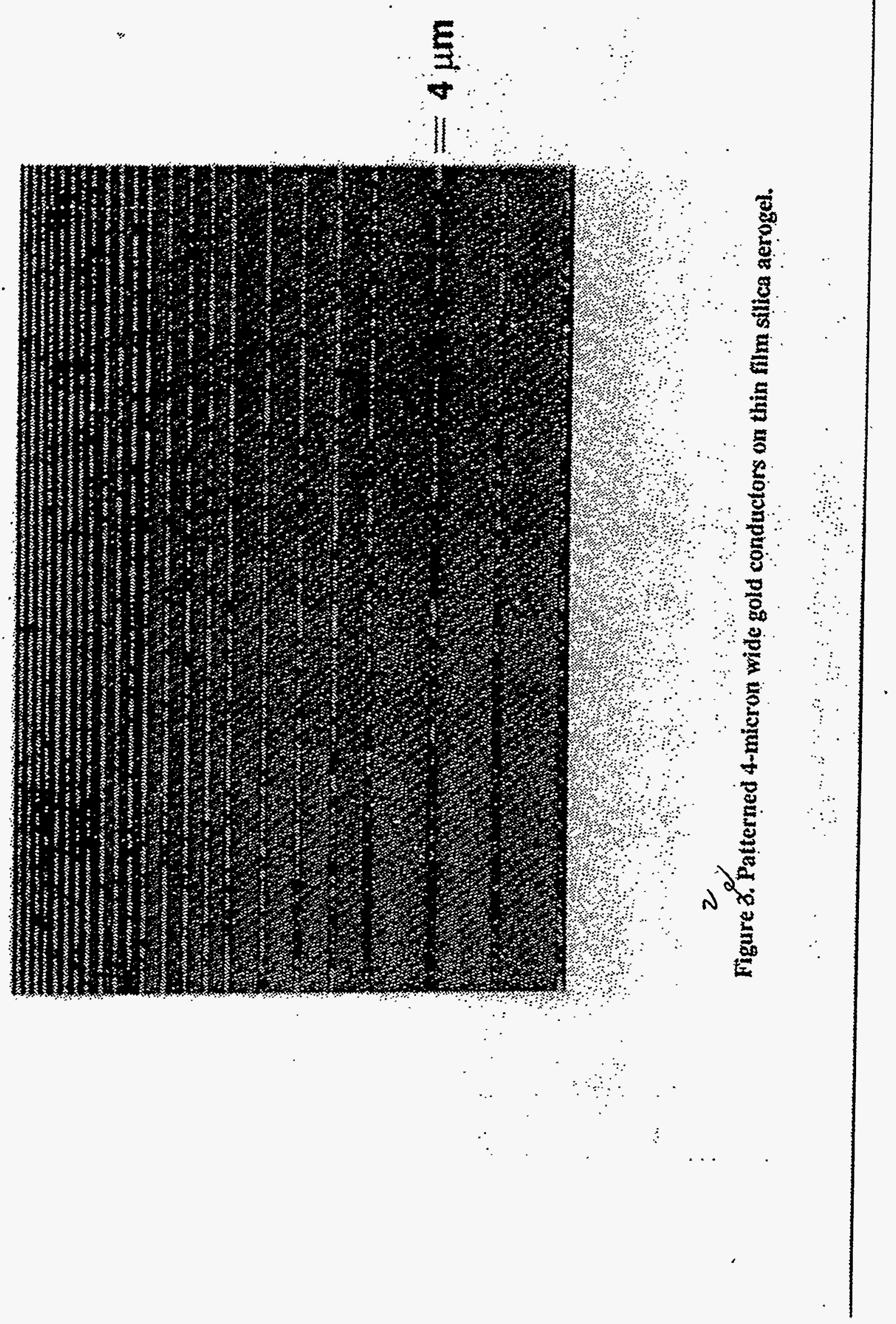



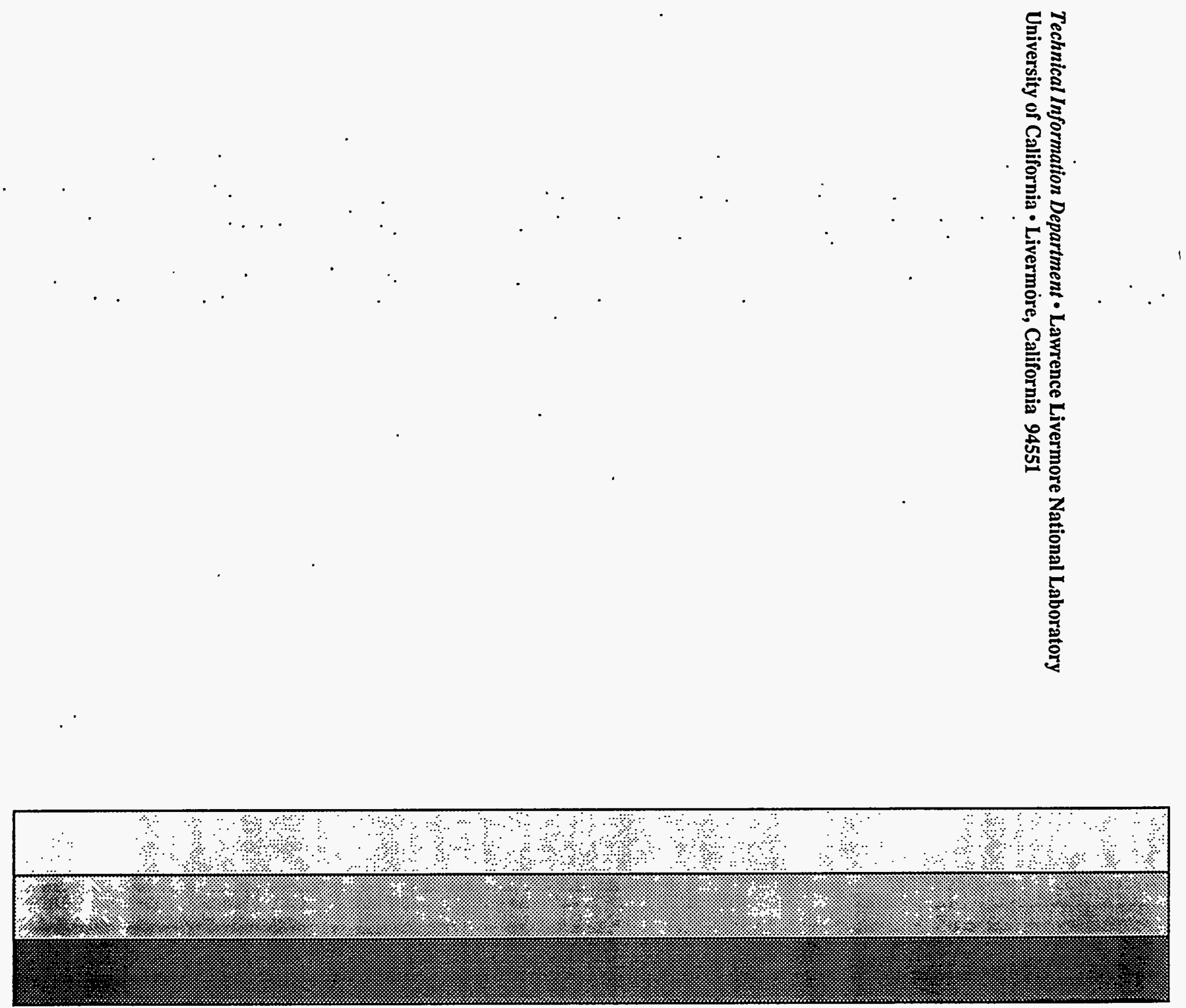